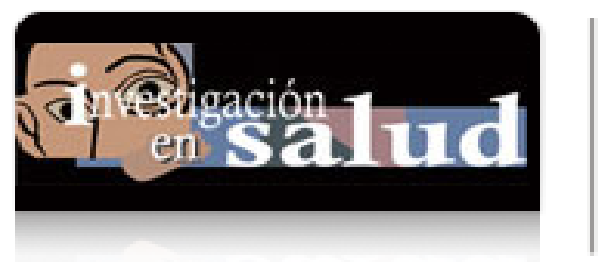

Investigación en Salud

ISSN: $1405-7980$

invsalud@cucs.udg.mx

Centro Universitario de Ciencias de la Salud México

Aldrete Rodríguez, María Guadalupe; Pando Moreno, Manuel; Aranda Beltrán, Carolina; Balcázar

Partida, Nidia

Síndrome de Burnout en maestros de educación básica, nivel primaria de Guadalajara

Investigación en Salud, vol. V, núm. 1, abril, 2003, p. 0

Centro Universitario de Ciencias de la Salud

Guadalajara, México

Disponible en: http://www.redalyc.org/articulo.oa?id=14200103

- Cómo citar el artículo

- Número completo

- Más información del artículo

- Página de la revista en redalyc.org

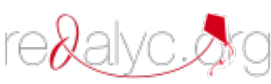

Sistema de Información Científica

Red de Revistas Científicas de América Latina, el Caribe, España y Portugal

Proyecto académico sin fines de lucro, desarrollado bajo la iniciativa de acceso abierto 


\title{
INVESTIGACIÓN EN SALUD
}

\section{Síndrome de Burnout en maestros de educación básica, nivel primaria de Guadalajara}

\section{Resumen}

\author{
Maria Guadalupe Aldrete Rodríguez. \\ Manuel Pando Moreno. \\ Carolina Aranda Beltrán \\ Nidia Balcázar Partida
}

\begin{abstract}
Los autores buscan identificar la prevalencia del Síndrome de Burnout en maestros de Educación Primaria de la Zona Metropolitana de Guadalajara y su relación con las actividades propias de su labor docente.

Es un estudio observacional, transversal y descriptivo en una muestra de maestros que laboraban frente a grupo en 25 escuelas primarias seleccionadas aleatoriamente de Guadalajara. La unidad de análisis fue el Síndrome de Burnout y su relación con las actividades que realizan como docentes. Para la captación de la información se utilizó un formulario autoaplicado que contenía las variables de interés, así como la Escala de Maslach Burnout Inventory.
\end{abstract}

Se estudiaron a 301 maestros. Las siguientes variables demostraron evidencia del Síndrome de quemarse en los maestros investigados: altos niveles de agotamiento emocional (25.9 por ciento), baja realización en su trabajo (21.6 por ciento) y altos niveles de despersonalización (5.6 por ciento). Sólo un 20.6 por ciento no presentan alteración en las áreas que evalúa la escala de Maslach, no se encontró relación con actividades como: planeación docentes, calificación de pruebas y tareas, elaboración de material didáctico. Un $80 \%$ de los docentes presentan el síndrome de quemarse por el trabajo y este no se relaciona con las actividades propias de su labor profesional.

Palabras clave: Síndrome de Burnout, docentes, educación básica.

\begin{abstract}
Abstrct
The authors search identification of the prevalence of Burnout Syndrome in grammar school teachers in the Guadalajara metropolitan area and its relationship to activities involved in teaching.

This is an observational, cross-sectional, and descriptive study of sample of classroom teachers in 25 Guadalajara grammar schools selected at random. The analysis focused on Burnout Syndrome and its relationship to teaching activities. Data was gathered via a questionnaire completed by each teacher, wich contained the variables under study, as well as the Maslach Burnout Inventory ( $\mathrm{MBI})$.
\end{abstract}

The study covered 301 teachers. The following variables demonstrated evidence of Burnout Syndrome in the teachers surveyed: high levels of emotional exhaustion ( $25.9 \%$ of teachers surveyed), low achievement at work (21.6\%), and high levels of depersonalization (5.6\%). Only $20.6 \%$ of the teachers surveyed did not show presence of Burnout Syndrome in the areas evaluated by the $\mathrm{MBI}$, and no relation was found with such activities as: planning of teaching activities, grading of test and homework, and development of teaching materials.

$80 \%$ of the teachers showed evidence of Burnout Syndrome in their work, but Burnout Syndrome was not related to activities involved in teaching.

Keywords: Burnout syndrome, teachers, basic education. 


\section{INVESTIGACIÓN EN SALUD}

\section{I ntroducción}

La función de docente en las escuelas primarias es depositaria social de una función determinante, contribuir a asegurar que los niños y jóvenes reciban una educación de calidad, que los forme como ciudadanos. Se trata de una profesión que requeriría de entrega, idealismo y servicio a los demás.

La educación básica de calidad guarda correlación con la posibilidad de desarrollo de un país (1), por lo que se debe buscar que el maestro tenga los elementos necesarios para cumplir con su función.

La docencia, como puesto de trabajo, obliga a la realización, a veces simultanea, de un sin fin de actividades, que van desde el cuidado y mantenimiento de la escuela, planeación de actividades docentes, elaboración de materiales didácticos, entre otras, y al mismo tiempo mantener constantes relaciones con: padres de familia, autoridades, compañeros y alumnos, lo que implica un duro esfuerzo, una significativa carga psíquica en el trabajo.

Ejemplo de ello es que el maestro, con una limitada libertad para desempeñar su trabajo tras la puerta cerrada de su aula, padece, al mismo tiempo, un cierto aislamiento para enfrentar su trabajo y para encontrar la solución a los numerosos problemas que se presentan en su quehacer cotidiano. En estas condiciones de trabajo, es común que los docentes puedan llegar a desencadenar un cierto grado de estrés (2), complicado generalmente con otros factores psicosociales negativos que acostumbran estar presentes en el ejercicio de la docencia, como sería el trabajo que realizan en casa, el comportamiento de los alumnos, la falta de promociones, las condiciones laborales insatisfactorias, entre otros (3).

En una revisión de 71 artículos realizada por Hiebert y Farber(1984)(4), encontraron evidencias para considerar a la docencia como una profesión estresante. La prevalencia del estrés y la respuesta a éste varia de maestro a maestro, dependiendo de las estrategias de enfrentamiento que utilice $(5,6,7,8)$.

Un bajo o mal funcionamiento de las estrategias implementadas, pueden hacer crónica la presencia del estrés y manifestarse el Síndrome de Burnout o Síndrome de Quemarse por el Trabajo. Este Síndrome ocupa un lugar destacado dentro de los riesgos laborales de carácter psicológico en personas que trabajan con personas $(9,10,11)$.

El Síndrome de Burnout es una de las principales causas de deterioro en el trabajo, cuyas consecuencias se manifiestan a nivel individual: en el plano de lo psicológico como enfado, ansiedad, agresividad verbal o física, desaliento, miedo, enojo, aislamiento; en el área física existen manifestaciones como fatiga, problemas gastrointestinales, cardiacos, o trastornos psicosomáticos; y en el nivel organizacional, con trastornos debidos a las actitudes negativas hacia el trabajo o hacia las personas que atiende (en este caso los alumnos), problemas de adaptación al rol o actividad que desempeña, incumplimiento de la tarea, ausentismo laboral, accidentes laborales, pérdida de interés por la profesión (10) y que en el caso que nos ocupa, se puede manifestar con repercusión en el proceso de enseñar y la calidad de las relaciones interpersonales que se derivan del trabajo o aún en la familia(12).

Por todo lo anterior, decidimos identificar la prevalencia del síndrome de quemarse por el trabajo en los maestros de Educación Básica nivel primaria y su relación con las actividades que realiza tanto a nivel del aula como fuera de ella. 


\section{INVESTIGACIÓN EN SALUD}

\section{Material y métodos}

Se realizó un estudio observacional descriptivo y transversal, en una población de maestros que laboraban frente a grupo en escuelas de Educación Básica nivel primaria ubicadas en Guadalajara.

El tamaño de la muestra fue calculado tomando en cuenta una población de docentes de Guadalajara de 7808 registrados en la estadística básica del ciclo escolar 2000-2001 de la Coordinación de Planeación Educativa. Por desconocer como se comporta el evento a investigar se calculó la muestra con una prevalencia de .5, un error estándar de .05 y un nivel de confianza de $90 \%$. quedando la muestra constituida por 300 maestros

Para localizar a los docentes que participaron en este estudio se realizó un muestreo aleatorio simple en el que se seleccionaron escuelas primarias. De las escuelas visitadas se entrevistaron a todos los docentes que laboraban ahí, hasta completar el tamaño de muestra. Los criterios de inclusión fueron: que atendieran cualquier grado de educación primaria y que tuvieran como mínimo 6 meses de trabajo frente a grupo.

Para el estudio se utilizaron cinco grupos de variables: las sociodemográficas: edad, sexo, estado civil; las relacionadas con el trabajo: categoría profesional, antigüedad profesional, situación laboral, turno de trabajo; las relacionadas con la docencia como: actividades de planeación, elaboración de material didáctico, actividades del proceso de evaluación; las actividades de la vida cotidiana familiar: Entendidas éstas operativamente como las acciones que realizan dentro de su hogar y los apoyos que se reciben y el Síndrome de quemarse categorizado en: a) agotamiento emocional (E.E) caracterizado por la pérdida progresiva de energía, el desgaste, la fatiga, etc.; b) la despersonalización (D) manifestada por un cambio negativo de actitudes y respuestas hacia los demás con irritabilidad y pérdida de motivación hacia el trabajo; y c) la falta de realización personal (PA) con respuestas negativas hacia sí mismo y el trabajo $(9,11)$.

La captación de información se realizó por medio de un documentos estructurado en dos partes: una que contenía las variables relacionadas con antecedentes laborales y actividades que se realizan. Esta parte del formulario fue piloteado con maestros que laboran en escuelas primaria que no fueron seleccionadas para este estudio.

Otra parte del instrumento fue el Maslash Burnout Inventory ( $\mathrm{MBI}$ ), éste es un instrumento ya probado y con respuestas de opción múltiple, tipo Liker. El cual fue adaptado para los maestros y en el que sólo se sustituyó la palabra pacientes por la de alumnos, el resto del instrumento permaneció sin modificaciones. Se ha validado su confiabilidad, encontrando para agotamiento emocional, una alfa de Cronbach de .90, alfa de .76 para despersonalización y una alfa de .76 para realización personal en el trabajo (11).

El procesamiento de la información: Las preguntas abiertas se leyeron en varias ocasiones para visualizar las ideas centrales y de esta manera agrupar las respuestas, siguiendo los criterios de similitud.

El MBI es un instrumento con respuestas de opción múltiple, por lo que se procedió a realizar el conteo de la información. Toda la información fue procesada con ayuda del paquete estadístico Epi Info 6. 


\section{INVESTIGACIÓN EN SALUD}

\section{Análisis de la información}

Para las variables de tipo cuantitativo se obtuvieron frecuencias absolutas y relativas y medidas de tendencia central y como medida de dispersión la desviación estándar.

Con el fin de encontrar algunas variables asociadas al síndrome de quemarse se procedió a integrar grupos de docentes: Con síndrome del quemado y sin síndrome (maestros que presentaban 2 ó 3 áreas afectadas) y se relacionaron con algunas variables relacionadas con el trabajo.

\section{Resultados}

Se entrevistaron a 301 docentes que laboran frente a grupo en 25 escuelas seleccionadas aleatoriamente de Guadalajara.

Las edades oscilaron de los 24 a 69 años con un promedio de 46 años y una desviación estándar de 13.9 , el $79.7 \%$ pertenecían al sexo femenino y el 17.9 al masculino. El $76.7 \%$ eran casados, $18.6 \%$ solteros, $2 \%$ divorciados y el resto viudos, separados o madres solteras.

El 36.4\% habían cursado la Normal Básica, 42.5\% la Licenciatura de Maestro de Educación Primaria, $11.6 \%$ Normal Superior y $2.7 \%$ realizado estudios de Maestría y el resto tenía otros estudios de especialidad dentro de la Universidad Pedagógica.

Un 69.1\% actualmente no está estudiando y el resto estudia alguna maestría (5\%), licenciatura $(4.7 \%)$, computación $(4.7 \%)$ y actividades artísticas $(4.7 \%)$, tan solo una persona estudia doctorado.

Los maestros entrevistados son egresados de escuelas formadoras de docentes desde el año 1948 al 2000, el promedio lo hizo en el ciclo escolar 1978-1979. Tienen una antigüedad de 1 a 54 años de servicio con un promedio de 27 y una desviación estándar de 21.5

El $42.1 \%$ tiene sólo una jornada de trabajo dentro del área educativa, el $43.2 \%$ tiene dos jornadas de trabajo dentro del área educativa. Fuera del área educativa el $22.5 \%$ labora como comerciante o empleado. El $69.5 \%$ de los entrevistados realiza labores domésticas después o antes de sus jornadas de trabajo.

De los 301 docentes entrevistados el 52.5\% laboran en escuelas pertenecientes al subsistema federalizado, $42.9 \%$ en el subsistema estatal, $3.7 \%$ en el particular y hubo 3 docentes que no nos proporcionaron esta información.

El $83.14 \%$ no han pedido licencia en los últimos dos años, el $63.1 \%$ considera no necesitarla, el resto se dividió igual entre no desatender a su grupo y por no poder prescindir de su sueldo. De los que si han pedido licencia un $7.6 \%$ el motivo fue enfermedad, el $4.3 \%$ por asuntos personales, $1.6 \%$ por gravidez, o simplemente para descansar $1.3 \%$.

Sobre las actividades que realizan los maestros los fines de semana, tenemos que, el $36.2 \%$ lo dedica a su familia, el $31.6 \%$ realiza tareas propias de su labor profesional, el $21.3 \%$ hace trabajo doméstico y el resto lo dedican a su capacitación profesional y a su persona y dos personas no contestaron. 


\section{INVESTIGACIÓN EN SALUD}

Al cuestionarles sobre si el tiempo asignado para cumplir con los requerimientos que implica la labor docente era el suficiente, el $60.5 \%$ respondió que no, las causas de esto es porque surgen imprevistos $(9.2 \%)$; son muchos los contenidos y actividades (10.8\%); solo falta tiempo $(24.6 \%)$; por comisiones escolares $(2.5 \%)$; el resto dio otros motivos. Los que respondieron que si alcanzaban (34.6\%) fue porque dijeron planear su trabajo con anticipación o simplemente porque les parece suficiente.

Tomando en cuenta lo anterior podemos observar en la Tabla número I, que los docentes realizan una serie de actividades propias de su función como docentes fuera de la jornada laboral.

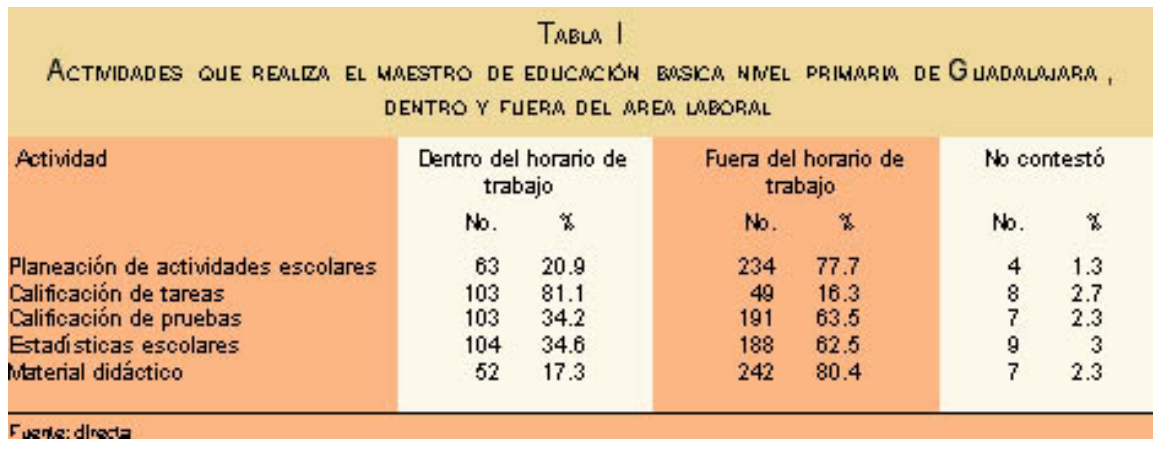

Al cuestionarles sobre las actividades que se realizan dentro del hogar, el $55.1 \%$ respondieron que las realizaban personalmente, el $21.9 \%$ dijeron que todos los miembros de la familia participaban, el $9.3 \%$ la ayuda se recibe de parte del cónyuge, el $7 \%$ contaban con una empleada doméstica, cuatro personas no contestaron y el resto dijeron que las actividades las hacen sus hijos u otro familiar.

Una de las preguntas realizadas sobre este punto fue la preparación de alimentos, en su mayoría es la persona entrevistada (maestra) quien lo realiza (55.5\%), sólo el $1.7 \%$ se dijo que es el padre, en el resto de los entrevistados las respuestas fueron que en esta actividad interactúan varios miembros de la familia con esta responsabilidad.

Dentro de cada familia siempre existen diferencias económicas que hacen que un miembro sea el que más aporta, el $51.8 \%$ de los entrevistados dijeron que era el cónyuge, el $36.2 \%$ es la madre la que más aporta, el $11.3 \%$ no contestaron, solo el $0.7 \%$ son los hijos .

En relación a los docentes que presentaron el síndrome del quemado, en alguna dimensión, en la tabla número II podemos observar que el $25.9 \%$ de los docentes presenta agotamiento emocional (EE) calificado como alto, el $21.6 \%$ de los docentes también fueron calificados en este mismo nivel en el aspecto de Baja realización en el trabajo y el $80 \%$ fueron evaluados como nivel bajo en el área de Despersonalización. 


\section{INVESTIGACIÓN EN SALUD}

\begin{tabular}{|c|c|c|c|c|c|c|}
\hline $\begin{array}{l}\text { PREVALEHCLO DEL SIHDR } \\
\text { IWVENT ORY (WEI) DOR }\end{array}$ & $\begin{array}{l}\text { OWE DE BUR } \\
\text { DIWENSNONES }\end{array}$ & $\begin{array}{l}\text { TABL } \\
\text { HOUT SEGUH } \\
\text { EN LOS WMES } \\
\text { GUMDML }\end{array}$ & $\begin{array}{l}\text { II } \\
\text { EScaLa DE } \\
\text { ROS DE ED } \\
\text { WMRA }\end{array}$ & $\begin{array}{l}\text { LORACOH D } \\
0 \mathrm{COH} \text { EASKC }\end{array}$ & $\begin{array}{l}\text { E M ASLOC } \\
\text { WWEL PRI }\end{array}$ & $\begin{array}{l}\text { BURHOUT } \\
\text { MaRla DE }\end{array}$ \\
\hline \multirow[t]{2}{*}{ Calificación } & \multicolumn{6}{|c|}{ Dimensiones del sindrome } \\
\hline & \multicolumn{2}{|c|}{$\begin{array}{l}\text { Agotamiento emocional } \\
\text { (EE) }\end{array}$} & \multicolumn{2}{|c|}{$\begin{array}{c}\text { Baja realiz ación en el } \\
\text { trabajo (PA) }\end{array}$} & \multicolumn{2}{|c|}{ Despersonaliz ación (D) } \\
\hline Bajo & 109 & 36.2 & 151 & 50.2 & 241 & 80.1 \\
\hline Medio & 114 & 37.9 & 85 & 28.2 & 43 & 14.3 \\
\hline Anto & 78 & 25.9 & 65 & 21.6 & 17 & 5.6 \\
\hline Total & 301 & $100 x$ & 301 & $100.0 \%$ & 301 & $100.0 \%$ \\
\hline
\end{tabular}

La tabla número III nos muestra las áreas en las que el docente se encuentra afectado y sólo el $20.6 \%$ de los docentes no presentan el síndrome de quemarse por el trabajo.

\begin{tabular}{|c|c|c|c|}
\hline \multicolumn{3}{|c|}{ 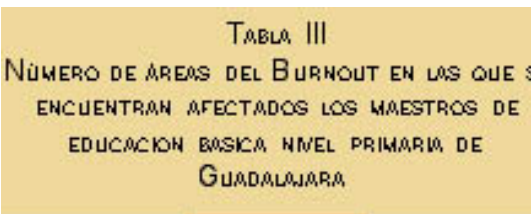 } & \multirow{6}{*}{$\begin{array}{l}\text { Después de realizar el análisis descriptivo se procedió } \\
\text { Eagrupar a los docentes según los resultados del MBI. Se } \\
\text { consideraron dos grupos. Un primer grupo formado por los } \\
\text { docentes que no presentaron ningun área afectada y como } \\
\text { segundo grupo los docentes que sí presentaron } \\
\text { problemática en alguna de la áreas que evalúa el MBI. Al } \\
\text { mismo tiempo las variables de trabajo se dicotomizaron, } \\
\text { por ejemplo: si el tiempo era considerado como suficiente } \\
\text { para la realización de las tareas o insuficiente, estado civil } \\
\text { casado o soltero, apoyo del hogar si/no etc. con los datos } \\
\text { de esta manera se procedió a realizar tablas de } 2 \times 2 \text { y se } \\
\text { utilizó la chi cuadrada. }\end{array}$} \\
\hline Areas & Frecuencia & Porcentaje & \\
\hline $\begin{array}{l}\text { Ninguna } \\
\text { Lha }\end{array}$ & & & \\
\hline & & & \\
\hline Tres & & & \\
\hline Total & 301 & 100.0 & \\
\hline
\end{tabular}

Encontramos que las variables que se relacionan con el Síndrome de quemarse son: la antigüedad, la escolaridad y trabajar en el subsistema federalizado, el no recibir apoyo para realizar las actividades del hogar y la antigüedad se asoció con el agotamiento emocional. Tabla número IV.

Al realizar el análisis considerando solo el sexo femenino se encontró asociación entre otras variables en el área de despersonalización como: el estado civil (casadas vs no casadas) $\mathrm{P}=.000$, el trabajar en más de una jornada, $\mathrm{P}$ $=.04$, así como realizar su actividad docente en el turno vespertino.

\section{Discusión}

La satisfacción en el trabajo, el éxito económico, y el reconocimiento de los demás constituyen, en general, lo menos que se espera obtener en un empleo.

TAELA IV

AsOClOCLNES ENCONTRMDas ENTRE LUS WRRLELES Y EL SIHOROWE DEB URHOUT EN LOS HMESTRCS DE EDUCACION EOSICA NMEL PRILARRL DE GuMDMLanaRma $p<0.05$

\begin{tabular}{|c|c|c|}
\hline Variable & $\mathrm{CHI}$ & $P$ \\
\hline $\begin{array}{l}\text { Agotaniento emocional y } \\
\text { antigüedad }\end{array}$ & 13.04 & .04 \\
\hline Escolaridad & 12.29 & .04 \\
\hline $\begin{array}{l}\text { Trabajar en el subsisterma } \\
\text { federaliz ado }\end{array}$ & 10.18 & .01 \\
\hline $\begin{array}{l}\text { Realiz ación de actividades del } \\
\text { hogar sin apoyo }\end{array}$ & 4.85 & .02 \\
\hline
\end{tabular}

De tal manera, la tarea de elegir una profesión y de hallar un trabajo adecuado donde ejercerla, produce una gran cantidad de tensión en las personas. Aún cuando una persona sepa claramente lo que desea hacer y posea la capacidad y el entrenamiento necesarios para llevarlo a cabo satisfactoriamente. 


\section{INVESTIGACIÓN EN SALUD}

El tiempo de ser docente al parecer puede producir altos niveles de ansiedad. La antigüedad se asoció al síndrome de quemarse, específicamente con la dimensión agotamiento emocional ¿Por qué el contacto diario con los alumnos hace que el docente se sienta emocionalmente agotado?, es una pregunta que requiere ser investigada.

Por otra parte los resultados nos mostraron que el $74 \%$ de los docentes consideran que el tiempo asignado para cumplir con todos los requerimientos que implica la labor docente no es el suficiente, aún así esta variable no se encontró asociada con el síndrome de quemarse, de igual forma el realizar actividades propias de la labor profesional como: Planear actividades, elaborar material didáctico, calificar pruebas y tareas, a pesar de que la mayoría de estas actividades las realiza fuera del horario establecido y fuera del área de trabajo.

Los maestros sin lugar a dudas tienen un sin fin de actividades que cumplir. Ha sido necesario ocupar parte de su tiempo de descanso en obligaciones propias de su trabajo, pues sus cinco horas que abarca su jornada laboral no le son suficientes. No existe límite para trabajar. Sus días de trabajo no solo abarcan de lunes a viernes sino hasta sábados y domingos si es necesario.

Una de las variables en las que sí se encontró asociación con el síndrome de quemarse es el hecho de laborar dentro del subsistema federalizado, tal vez el docente se encuentre con mayores exigencias para realizar su trabajo. Esto podría deberse a las administraciones de dicho subsistema, en el que puedan existir elementos de presión. Esta variable podría ser objeto de estudio de investigaciones futuras, analizando otra serie de variables en cuanto a la organización del trabajo o las presiones a las que se encuentran sometidos los docentes para cumplir sus actividades o a los mecanismos o estrategias que se utilizan para que los maestros realicen su trabajo.

A mayor escolaridad se asoció al Síndrome de quemarse por el trabajo, la teoría de Schaufeli en la que el docente al estar más preparado académicamente percibe por este hecho que tendría más posibilidades de promoción profesional y al no lograrlo puede afectar su autoestima y sus relaciones laborales y esto le puede estar generando el síndrome. Algunos docentes tienen posibilidades de ingresar a la carrera magisterial, pero el beneficio es solo en el plano económico, no hay una movilidad, no hay posibilidades de ascender, continuando como docente de nivel primaria; tal vez esto es lo que está haciendo que el docente presente esta problemática. El $80 \%$ de los docentes son maestras y éstas realizan un sinfín de actividades dentro del hogar, cuando no cuentan con apoyo para realizarlas se encuentran con el riesgo de quemarse. En la actualidad con la creciente participación de la mujer en el ámbito laboral se ha ido aumentando la participación de todos los miembros de la familia dentro de las labores domésticas, pero aún así, es la mujer en quién recae la mayor parte de la responsabilidad del hogar. Los mayores problemas de las mujeres en su vida cotidiana, proviene de la división de su tiempo entre las múltiples tareas que realizan (13). La mujer que trabaja y que no prescinde de sus roles sociales, cumple una doble jornada (14) aumentando así sus niveles de estrés. Los quehaceres diarios de una ama de casa lejos de resultar creativos o estimulantes son causa en numerosas ocasiones de angustias y obsesiones (15) que repercuten en estrés crónico como lo constatamos en nuestros resultados. La presión de las tareas hogareñas nunca se olvida y, si conjuntamos esto con la carga de trabajo de ser docente se podía pensar que ésta tiene mayor posibilidad para presentar el Síndrome de quemarse.

Los argumentos reflejados en la literatura existentes para explicar la variable estado civil, han encontrado algún tipo de relación significativa con el síndrome del quemarse por el trabajo en los sujetos solteros con índices mayores que en los sujetos casados, en diversas profesiones. Al realizar un análisis por género encontramos que las maestras presentan índices más altos, tanto de agotamiento emocional como de baja realización en el trabajo en aquellas que están casadas. Lo que nos hace pensar que esta variable influye de una manera determinante en la presencia del Burnout, aunque no de una manera aislada, pues pensamos que lo que influye en el proceso de quemarse por el trabajo más que ser el estado civil son las responsabilidades que se tienen dentro del hogar y con frecuencia se realizan sin el apoyo emocional de parte de los familiares 


\section{INVESTIGACIÓN EN SALUD}

(esposo) o por la calidad de las relaciones conyugales y familiares. Greenglass (1989)(16), en una muestra con maestros obtuvo que los sujetos con menos satisfacción matrimonial expresaban más sentimientos de estar quemados por el trabajo.

La relación con los niveles que presentan las docentes de despersonalización de acuerdo al número de jornadas que tienen es otra variable de vital importancia en nuestra investigación, al continuar con el análisis por género encontramos que las maestras que trabajan dos turnos están más afectadas.

Los resultados que se obtuvieron fueron valiosos ya que la mayoría de maestras tienen una doble ocupación: trabajan doble plaza en el magisterio, estudian o bien tienen otro trabajo además de la docencia en primarias. Creemos que el tener una doble jornada provoca en las maestras estar tan enfrascadas en su labor que no dedican el tiempo suficiente a su vida personal $y$ a sus relaciones familiares teniendo una falta de sentimientos afectivos, endureciéndose emocionalmente y actuando en ocasiones deshumanizadas hacia las personas con que trabaja (compañeros, superiores, alumnos, padres de familia) siendo estas características distintivas de la despersonalización.

\section{Referencias bibliográficas}

1. Arnaut A. La federalización educativa en México 1989-1994. México,SEP: 1998. 305 págs.

2. Lortie D. Los límites de la socialización del maestro. En: Rockwell E. Ser maestro, estudios sobre el trabajo docente. México, El caballito; 1985. 37 págs.

3. Travers C., Cooper C. El estres de los profesores, la presión en la actividad docente, España, Paidós. 1997: 17 págs.

4. Hiebert B.A., Farber I. Teacher Stress: literature Survey with a few Surprises. Canadian Jounal of education. 1984;9(1):14-27

5. Tunnelclife M.R., Leach D.J. Relative efificacy of using behavioral consultation as an approach to teacher stress management. Journal of School Psycology. 1986;24:123-131.

6. Dunham J. Coping with stress in school, Special Education. Forward Trends. 1983; 10(2):6-9.

7. Pratt J. Perceived stress among teachers: The efects of age and Background of children. Taugh Educaional Review. 1978; 30: 3-14.

8. Hawkes R.R., Dedrick C.V. Teacher stress: Phase II of a Descriptive Study Nacional Association of secondary School. Principals Bulletin. 1983;67(461):78-83.

9. Maslach C., Jackson S.E. Maslach Burnout Inventory. Palo Alto California, Consulting Psychologists; 1986.

10. Gil P., Peiró J.M. Desgaste psíquico en el trabajo: el síndrome de quemarse. España, Síntesis Psicológica: 1997.

11. Gil-Monte P. R. Validez factorial de la adaptación al español del Maslach Burnout InventoryGeneral Survery. Salud Pública de México. 2002;44(2):33-40.

12. Ramos F. El Síndrome de Burn-out en los universitarios. Revista Vivat Academia. 2003: 5(41). www2.uah.es/vivatacademia/congreso.gtm 


\section{INVESTIGACIÓN EN SALUD}

13. García B., Muñoz O. Trabajo femenino y de vida formal en México. México, El Colegio de México: 1994. 30, 31, 42, 43, 56, 57, 106, 107, 112, 115 págs.

14. CIESAS. Mujeres y Sociedad (salario, hogar y acción social en el occidente de México). El Colegio de Jalisco, CIESAS: 1998. 12,13,18 págs.

15. Catalá M. El cuerpo de la psicología femenina. Barcelona, INDIGO: 1991. 61-65 págs.

16. Greenglass E., Burke RJ. Career orientations anda career development among male and female teachers. Psychol Report 1989; 64-59:591-598.

Dra. Maria Guadalupe Aldrete Rodríguez.

Dr. Manuel Pando Moreno.

MCSP. Carolina Aranda Beltrán

Dra. Nidia Balcázar Partida

Investigadores del Instituto Regional de Investigación en Salud Pública.

Departamento de Salud Pública.

Universidad de Guadalajara, México.

Correspondencia:

Dra. Ma. Guadalupe Aldrete Rodríguez

Domicilio: CUCS/Depto Salud Pública/IRISP

Tel.: 36-17-99-35

Artículo con "Conflicto de interés no declarado" 